\title{
Navajo Language
}

National Cancer Institute

\section{Source}

National Cancer Institute. Navajo Language. NCI Thesaurus. Code C154045.

A Southern Athabaskan language of the Na-Dene family spoken primarily in the Southwestern United States, especially in the Navajo Nation. 